\title{
Incomplete right bundle branch block: Challenges in electrocardiogram diagnosis
}

\author{
Mariana Floria1, 2 (1), Alexandra Noela Parteni1, 2 (D, Ioana Alexandra Neagu2, 3 (1), Radu Andy Sascau², 3 (D), \\ Cristian Statescu², 3 (iD, Daniela Maria Tanase2, 4 (D) \\ ${ }^{1}$ Internal Medicine Clinic, “Dr. Iacob Czihac” Military Emergency Clinical Hospital; Iasi-Romania \\ ${ }^{2}$ Department of Internal Medicine, "Grigore T. Popa" University of Medicine and Pharmacy; Iasi-Romania \\ 3“Prof. Dr. George I.M. Georgescu” Institute of Cardiovascular Disease; Iasi-Romania \\ ${ }^{4}$ Internal Medicine Clinic, "St. Spiridon" County Clinical Emergency Hospital; Iasi-Romania
}

\section{ABSTRACT}

Incomplete right bundle branch block (IRBBB), an entity undefined by a general consensus, can express a large pallet of both benign and pathological patterns. IRBBB is a common electrocardiogram (ECG) finding at all ages, more frequent in men and athletes. Usually, IRBBB does not need further evaluation; however, if abnormalities are found on the clinical exam, heart disease should be excluded. The RSR' pattern and a QRS width below $100 \mathrm{~ms}$ define the crista supraventricularis (CSV) pattern. CSV is a right ventricular crest, one of the last structures to be depolarized by the Purkinje network. CSV pattern might result from posterior apex deviation, subpulmonic area delay, or late CSV activation. IRBBB can appear because of higher placement of electrodes V1 and V2 and pectus excavatum, in which P wave is negative, or in athletes, considered a benign pattern unless family history, symptoms, or left ventricular hypertrophy. It is necessary to differentiate IRBBB from pathological patterns such as type-2 Brugada ECG pattern, right ventricular enlargement, arrhythmogenic right ventricular cardiomyopathy, ventricular preexcitation-Wolf-Parkinson-White syndrome, and hyperkalemia. Examiners should be particularly alert to the splitting of the second heart sound because RBBB is a common finding in ostium secundum atrial septal defect. Therefore, clinicians need to be familiar with this ECG finding, which is not always a benign condition.

Keywords: bundle branch block, ECG, Brugada syndrome, Wolf-Parkinson-White syndrome

Cite this article as: Floria M, Parteni AN, Neagu IA, Sascau RA, Statescu C, Tanase DM. Incomplete right bundle branch block: Challenges in electrocardiogram diagnosis. Anatol J Cardiol 2021; 25: 380-4.

\section{Introduction}

Right bundle branch block (RBBB) represents an abnormality in the intraventricular electrical conduction system of the human heart, generating enlarged QRS and modified vectors on the electrocardiogram (ECG) (1). It appears with a prevalence of $3 \%$ to $7 \%$ in the general population, more frequently in men than in women. (2).

\section{Definition}

According to the American Heart Association/American College of Cardiology Foundation/Heart Rhythm Foundation (AHA) ACCF/HRS) recommendations for the standardization and interpretation of the ECG, incomplete RBBB (IRBBB) is defined by:
1. QRS duration between 110 and $120 \mathrm{~ms}$ in adults.

2. rsr, rsR, or rSR in leads V1 or V2. The R or r deflection is usually wider than the initial $R$ wave. In a minority of patients, a wide and often notched $\mathrm{R}$ wave pattern may be seen in leads V1 and/or V2.

3. S wave of greater duration than $\mathrm{R}$ wave or greater than $40 \mathrm{~ms}$ in leads I and V6 in adults.

4. Normal $\mathrm{R}$ peak time in leads $\mathrm{V} 5$ and $\mathrm{V} 6$ but greater than $50 \mathrm{~ms}$ in lead V1.

The first 3 criteria should be present to make the diagnosis (Fig. 1a). When a pure dominant $R$ wave with or without a notch is present in V1, criterion 4 should be satisfied (1). Although we reproduced the AHA/ACCF/HRS recommendations; in fact, there is no consensus in the literature regarding IRBBB definition (2). 


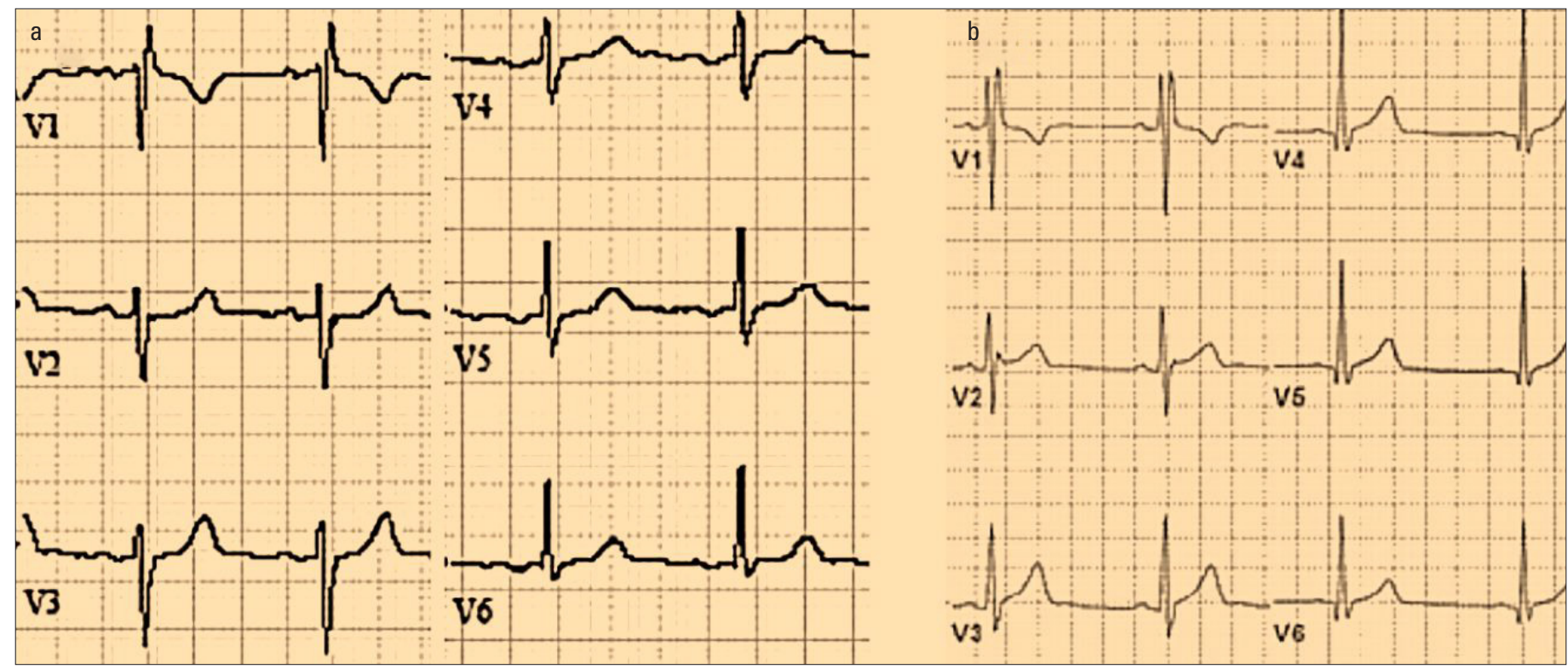

Figure 1. a. Incomplete right bundle block pattern. b. Crista supraventricularis pattern

\section{IRBBB patterns}

A QRS width below $100 \mathrm{~ms}, \mathrm{~S}$ wave $<40 \mathrm{~ms}$ in I or $\mathrm{V} 6$ together with an RSR' pattern in lead V1 (Fig. 1b) define the crista supraventricularis (CSV) pattern. $(3,4)$. CSV is a crest originated from the ventricular muscle (3), attachment from the right ventricular free wall to the central upper portion of the interventricular septum (5), between the tricuspid and pulmonic valve (3). The CSV pattern appears when the muscular structure separating anterosuperior tricuspid leaflet from the pulmonary valve is compressed, and with it the connection between the Purkinje network and the septal network. It is more frequent in septal defects or right ventricular hypertrophy and is partially resolved after surgical correction, showing the connection with right ventricular overload (6).

CSV is fully supplied by the Purkinje network and is one of the last structures to be depolarized (3). A small membranous septum implies a close proximity between the right bundle branch and CSV where it unites the interventricular septum. It is almost entirely fed from the proximal right coronary artery passing directly over it (5).

CSV pattern might result from posterior apex deviation, subpulmonic area delay, or late CSV activation. The mechanisms for CSV pattern occurrence seem to differ from those causing IRBBB. The differentiation between these 2 ECG manifestations may prevent misdiagnosis of actual CSV pattern as IRBBB, allowing a better electrocardiographic characterization in athletes (3).

In a study conducted on young athletes showed a higher prevalence of CSV patterns (13.3\%) than IRBBB (8.6\%); an important difference given that IRBBB can be associated with some serious conditions such as arrhythmogenic right ventricular cardiomyopathy, Brugada syndrome, and atrial septal defect (4).

\section{Challenges in IRBBB interpretation}

IRBBB in athletes was formerly considered a benign pattern (7) that did not need further investigation unless family history or relevant symptoms or signs of left ventricular hypertrophy were present (8). IRBBB can also appear owing to higher placement of electrodes V1 and V2 and pectus excavatum in which $\mathrm{P}$ wave is negative (7).

For patients with heart disease, a link has been found between IRBBB and the increase in all-cause mortality, meaning that IRBBB may be more than just a benign finding, being associated with age related degenerative heart disease, overload of the right ventricle, right ventricle enlargement, and left ventricular remodeling, a physiological exercise-induced outcome. Therefore, IRBBB may have a different meaning depending on the age of the patient: youth or elderly (9).

It is also necessary to differentiate from pathological patterns such as type-2 Brugada ECG pattern, right ventricular enlargement, ventricular preexcitation [Wolf-Parkinson-White (WPW) syndrome, Fig. 2], and hyperkalemia (7).

\section{Right ventricular enlargement}

Right ventricular hypertrophy or dilatation causes a delay in activation of some regions (7). Electrogram-based studies have shown that IRBBB is not always caused by an abnormality along the right bundle branch or in the right peripheral Purkinje system, but may be a result of increased right ventricular free wall thickness (10).

A delay in activation owing to right ventricular enlargement can be caused by a large pallet of pathologies, accompanied by specific ECG findings.

One of them is mitral valve disease, associated with pulmonary hypertension, with $\mathrm{P}$ wave enlargement. In congenital pul- 


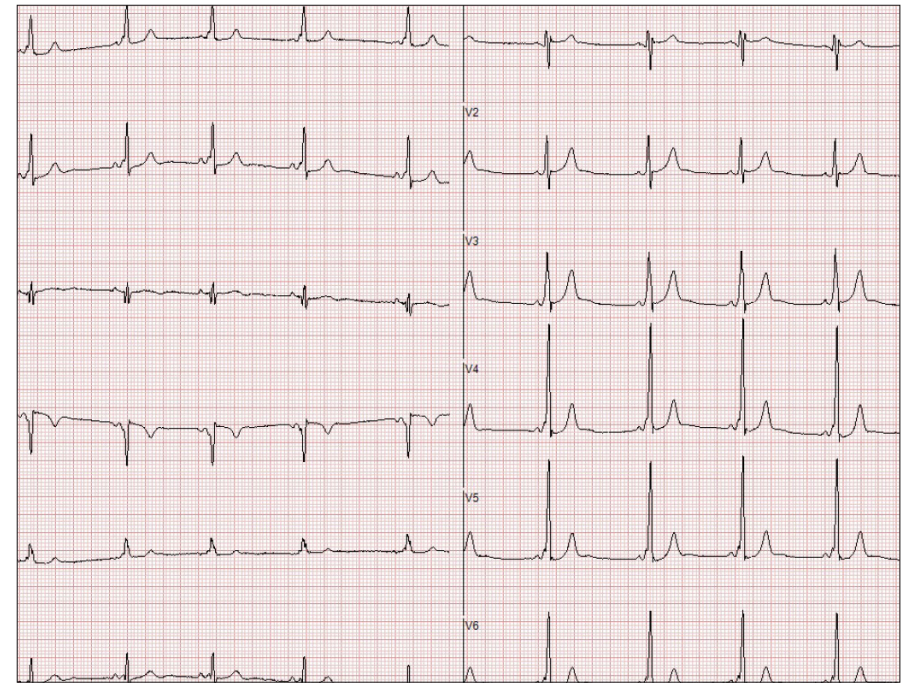

Figure 2. Ventricular preexcitation-Wolf-Parkinson-White syndrome

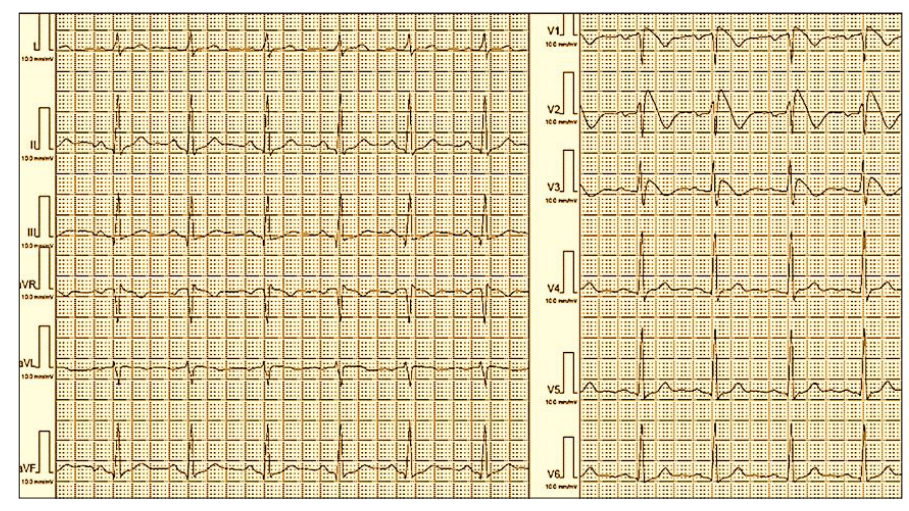

Figure 3. Type 1 Brugada syndrome

monary stenosis, there is no $\mathrm{P}$ wave enlargement, and the $\mathrm{r}^{\prime}$ is lower than r. Chronic obstructive pulmonary disease has an $\mathrm{rSr}^{\prime}$ pattern, V1 lead has a low voltage, and the $\mathrm{P}$ wave has no negative component. Ebstein disease has a peculiar $\mathrm{P}$ wave and a RBBB with $R^{\prime}$ bigger then $r$ (7).

Hyperkalemia is recognized by its tall, wide, and peaked T wave, with an inconstant abrupt ST elevation (7).

\section{Arrhythmogenic right ventricular cardiomyopathy}

During exercise, the cardiac output increases. If the systemic circulation accommodates through vasodilatation, pulmonary vascular system is less compliant, thus generating high pulmonary artery pressure. Normally, a healthy right ventricle adapts to this stress, but high intense exercise can cause transient myocardial fatigue, expressed through decreased right ventricular function, oversized chamber, and increased cardiac biomarkers. Repeated high intensity exercise can manifest similar to an arrhythmogenic cardiomyopathy with huge right ventricular dilatation, ventricular arrhythmias, and reduced ejection fraction (11).

Because of the presence of T-wave inversion, possibly associated with IRBBB, and because the epsilon wave is sometimes confused with an $r^{\prime}$ wave (11), arrhythmogenic right ventricular cardiomyopathy may be suspected, although "typical" IRBBB is uncommon in patients with arrhythmogenic right ventricular cardiomyopathy. When the IRBBB is associated with T-wave inversion extending beyond V2, including leads V3 and V4 or is associated with premature ventricular beats with a left bundle branch block morphology, arrhythmogenic right ventricular cardiomyopathy should be taken into suspected (8).

\section{Brugada syndrome}

RBBB needs to be distinguished from Brugada syndrome and its ECG appearance: J-wave, a mild positive deflection at the R-ST junction, in leads V1 and V2, with no changes in other leads (8).

To differentiate Brugada syndrome from RBBB, the J-point elevation and a high take-off ST-segment, which reflect early repolarization and not a delayed right ventricular activation specific to RBBB can be considered. The descending ST-segment is followed by a coved type or a saddle-back type T wave (8).

If type I (coved pattern, Fig. 3) is easily recognized, type II (saddle-back pattern that includes former II and III types) points to a different diagnosis. Although $r^{\prime}$ wave is peaked in IRBBB, in Brugada type II it is rounded and lower. Furthermore, in Brugada syndrome, there is a mismatch between QRS duration in right precordial leads, wider than the lateral ones, which is absent in IRBBB (7).

Corrado index, defined as ST elevation at high takeoff of QRS/ST elevation at $80 \mathrm{~ms}$ is $>1$ in type 1 Brugada pattern and $<1$ in athletes (7).

In RBBB, the $\mathrm{R}^{\prime}$ wave in $\mathrm{V} 1$ and $\mathrm{V} 2$ has a correspondent $\mathrm{S}$ wave in leads I and V6 and no ST elevation in the right precordial leads. For a clear diagnosis, a pharmacological test with sodium channel blockers may be necessary (8).

\section{WPW syndrome}

In WPW syndrome (Fig. 4), the subtle preexcitation is challenging. There is a RS morphology in V1, V2 with negative ORS in the inferior leads (III and aVF) and $-30^{\circ}$ to $-90^{\circ}$ ORS axis in posteroseptal accessory pathways. Moreover, it can present as a high $\mathrm{R}$ wave ( $\mathrm{RSr} r^{\prime}$ ) in the same $\mathrm{V} 1, \mathrm{~V} 2$ leads with negative morphology in lateral leads $(\mathrm{I}, \mathrm{aVL})$ with $+90^{\circ}$ up to $+150^{\circ}$ axis in left lateral accessory pathways (7).

\section{Tips and tricks in IRBBB diagnosis}

Examiners should be particularly alert to any symptom, especially splitting of the second heart sound, because RBBB typically accompanies atrial septal defect such as ostium secundum (8).

The ECG in an atrial septal defect shows an rSr' or rsR' morphology that mirrors right ventricular overload rather than a conduction delay in the right bundle branch. The $r$ is often enlarged and hazy, and $R$ wave presents a notch in the inferior leads. Onethird of the patients have right atrial enlargement. Pulmonary hypertension associates with right axis deviation (7). 


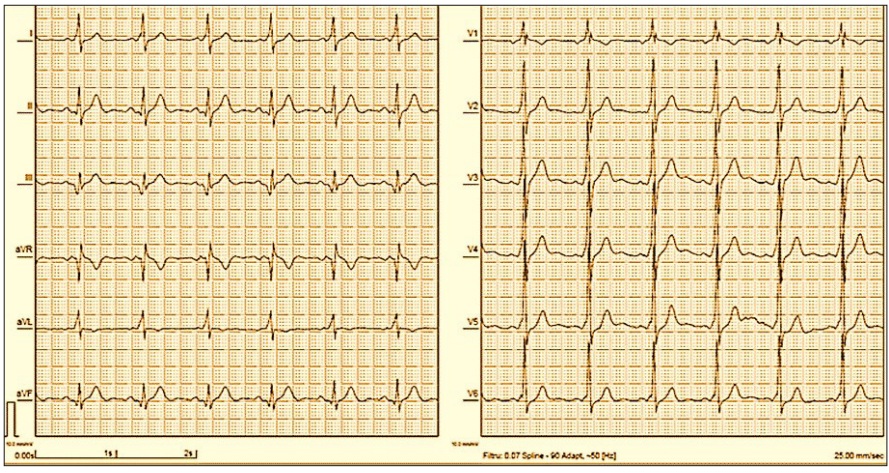

Figure 4. Ventricular preexcitation syndrome with $\mathrm{RSr}^{\prime}$ aspect in $\mathrm{V} 1$

There are pathologies with no structural abnormalities, like Brugada syndrome and WPW syndrome, in which electrocardiography and echocardiography alone are not enough; and other diagnostic and management options as well as some precautions should be taken.

In Brugada syndrome, the management options have a weak level of evidence because of its low incidence. There are certain drugs that should be avoided like some antiarrhythmic drugs (ajmaline, allapinin, ethacizin, flecainide, pilsicainide, procainamide, and propafenone), psychotropic drugs (amitriptyline, clomipramine, desipramine, lithium, loxapine, nortriptyline, oxcarbazepine, and trifluoperazine), anesthetics/analgesics (bupivacaine, procaine, and propofol), and other substances (acetylcholine, cannabis, cocaine, and ergonovine). Along with the avoidance of these drugs, Class 1 (IC) recommendations, include prompt treatment of fever and the avoidance of excessive alcohol intake and large meals (12).

Therapeutic options include ICD (implantable cardiac defibrillator), quinidine or isoproterenol, and even catheter ablation in patients with a history of electrical storms or repeated appropriate ICD shocks, which requires programmed ventricular stimulation.

Genetic screening can detect genes associated with Brugada syndrome; however, the results do not currently influence prognosis or treatment (12).

In WPW syndrome, electrophysiological study with ablation is needed in patients resuscitated from aborted cardiac arrest because of atrial fibrillation and rapid conduction over the accessory pathway causing ventricular fibrillation. Ablation also should be considered if the patient is symptomatic (e.g. with syncope or palpitations), and/or the refractory period of the accessory pathway is $\leq 240 \mathrm{~ms}$. Treatment with calcium antagonists (verapamil) or digoxin should be avoided (12).

Other tips to distinguish an IRBBB from a normal $r S r^{\prime}$ are the following findings on right precordial leads (V1, V2): diminution of the $S$ wave depth, inversion of ratio of the S wave depth to SV1/ SV2, slurring of the downstroke or upstroke of the $S$ wave, and prolongation of the QRS duration to $\geq 0.10 \mathrm{~s}$ (13).

\section{IRBBB significance and prognosis}

IRBBB is common in all ages, being studied in school children and adults, and it is not related to increased echocardiographic findings or cardiovascular mortality $(14,15)$. Classic IRBBB usually is benign; but if abnormalities are found on the clinical exam, any kind of heart disease should be excluded (15).

IRBBB associated with horizontal or inverted proximal T wave limb in the right precordial leads has a positive predictive value in diagnosing an atrial septal defect (16). Clinicians also should pay attention to interpreting signal-averaged electrocardiograms with IRBBB because the delayed terminal conduction may cause false positive late potentials (17).

It seems that IRBBB is strongly and independently associated with early-onset lone atrial fibrillation (18).

Regarding extracardiac association, one-third of patients with facioscapulohumeral muscular dystrophy present with IRBBB with no cardiomyopathy as an isolated finding, suggesting a selective involvement of His-Purkinje system (19).

\section{Conclusion}

To summarize, IRBBB is a common ECG finding in all ages, more frequent in men and athletes. With a negative personal and family history and normal physical exam, IRBBB does not need further evaluation as it is not associated with any harmful outcome. However, if abnormalities are found on the clinical exam, heart disease should be excluded.

Conflict of interest: None declared.

Peer-review: Externally peer-reviewed.

Author contributions: Concept - M.F., A.N.P.; Design - M.F., A.N.P.; Supervision - M.F., R.A.S., C.S., D.M.T.; Fundings - None; Materials M.F., A.N.P., I.A.N.; Data collection \&/or processing - R.A.S., D.M.T.; Analysis \&/or interpretation - C.S., D.M.T.; Literature search - R.A.S., C.S.; Writing - A.N.P., I.A.N.; Critical review - A.N.P., I.A.N.

\section{References}

1. Surawicz B, Childers R, Deal BJ, Gettes LS, Bailey JJ, Gorgels A, et al.; American Heart Association Electrocardiography and Arrhythmias Committee, Council on Clinical Cardiology; American College of Cardiology Foundation; Heart Rhythm Society. AHA/ ACCF/HRS recommendations for the standardization and interpretation of the electrocardiogram: part III: intraventricular conduction disturbances: a scientific statement from the American Heart Association Electrocardiography and Arrhythmias Committee, Council on Clinical Cardiology; the American College of Cardiology Foundation; and the Heart Rhythm Society. Endorsed by the International Society for Computerized Electrocardiology. J Am Coll Cardiol 2009; 53: 976-81. [Crossref]

2. Nielsen JB, Olesen MS, Tangø M, Haunsø S, Holst AG, Svendsen $\mathrm{JH}$. Incomplete right bundle branch block: a novel electrocardiographic marker for lone atrial fibrillation. Europace 2011; 13: 182-7. [Crossref]

3. Martinez-Sellés $M$, Diaz-Gonzalez L, Lucia A. Right ventricular remodeling in athletes and crista supraventricularis pattern. Clin Cardiol 2020; 43: 657. [Crossref]

4. Diaz-Gonzalez L, Bruña V, Velásquez-Rodriguez J, Valenzuela PL, Valero-Masa MJ, González-Saldívar H, et al. Young athletes' ECG: 
Incomplete right bundle branch block vs crista supraventricularis pattern. Scand J Med Sci Sports 2020; 30: 1992-8. [Crossref]

5. James TN. Anatomy of the crista supraventricularis: its importance for understanding right ventricular function, right ventricular infarction and related conditions. J Am Coll Cardiol 1985; 6: 108395. [Crossref]

6. Carro A, Sanz-de la Garza M, Caselli S. Reply: Right ventricular remodeling in athletes and crista supraventricularis pattern. Clin Cardiol 2020; 43: 658. [Crossref]

7. Baranchuk A, Enriquez A, García-Niebla J, Bayés-Genís A, Villuendas $\mathrm{R}$, Bayés de Luna $A$. Differential diagnosis of $r \mathrm{Sr}^{\prime}$ pattern in leads V1 -V2. Comprehensive review and proposed algorithm. Ann Noninvasive Electrocardiol 2015; 20: 7-17. [Crossref]

8. Corrado D, Pellic cia A, Heidbuchel H, Sharma S, Link M, Basso C, et al.; Section of Sports Cardiology, European Association of Cardiovascular Prevention and Rehabilitation. Recommendations for interpretation of 12-lead electrocardiogram in the athlete. Eur Heart J 2010; 31: 243-59. [Crossref]

9. Rankinen J, Haataja P, Lyytikäinen LP, Huhtala H, Lehtimäki T, Kähönen $M$, et al. Long-term outcome of intraventricular conduction delays in the general population. Ann Noninvasive Electrocardiol 2021; 26: e12788. [Crossref]

10. Moore EN, Boineau JP, Patterson DF. Incomplete right bundlebranch block. An electrocardiographic enigma and possible misnomer. Circulation 1971; 44: 678-87. [Crossref]

11. Sanz-de la Garza M, Carro A, Caselli S. How to interpret right ventricular remodeling in athletes. Clin Cardiol 2020; 43: 843-51. [Crossref]

12. Priori SG, Blomström-Lundqvist C, Mazzanti A, Blom N, Borggrefe M, Camm J, et al.; ESC Scientific Document Group. 2015 ESC Guidelines for the management of patients with ventricular arrhythmias and the prevention of sudden cardiac death: The Task Force for the Management of Patients with Ventricular Arrhythmias and the Prevention of Sudden Cardiac Death of the European Society of Cardiology (ESC). Endorsed by: Association for European Paediatric and Congenital Cardiology (AEPC). Eur Heart J 2015; 36: 2793-867. [Crossref]

13. Mauric AT, Samani NJ, de Bono DP. When should we diagnose incomplete right bundle branch block? Eur Heart J 1993; 14: 602-6. [Crossref]

14. Meziab 0, Abrams DJ, Alexander ME, Bevilacqua L, Bezzerides V, Mah DY, et al. Utility of incomplete right bundle branch block as an isolated ECG finding in children undergoing initial cardiac evaluation. Congenit Heart Dis 2018; 13: 419-27. [Crossref]

15. Bussink BE, Holst AG, Jespersen L, Deckers JW, Jensen GB, Prescott E. Right bundle branch block: prevalence, risk factors, and outcome in the general population: results from the Copenhagen City Heart Study. Eur Heart J 2013; 34: 138-46. [Crossref]

16. Wang MX, Wu GF, Gu JL, Li L, Lu K, Yang D, et al. Defective T wave combined with incomplete right bundle branch block: a new electrocardiographic index for diagnosing atrial septal defect. Chin Med J (Engl) 2012; 125: 1057-62.

17. Manolis AS, Chiladakis JA, Malakos JS, Vassilikos V, Maounis T, Cokkinos DV. Abnormal signal-averaged electrocardiograms in patients with incomplete right bundle-branch block. Clin Cardiol 1997; 20: 17-22. [Crossref]

18. Nielsen JB, Olesen MS, Tangø M, Haunsø S, Holst AG, Svendsen $\mathrm{JH}$. Incomplete right bundle branch block: a novel electrocardiographic marker for lone atrial fibrillation. Europace 2011; 13: 182-7. [Crossref]

19. van Dijk GP, van der Kooi E, Behin A, Smeets J, Timmermans J, van der Maarel $S$, et al. High prevalence of incomplete right bundle branch block in facioscapulohumeral muscular dystrophy without cardiac symptoms. Funct Neurol 2014; 29: 159-65. [Crossref] 